\title{
ON THE PRICING OF AMERICAN OPTIONS IN EXPONENTIAL LÉVY MARKETS
}

\author{
ROMAN V. IVANOV, ${ }^{*}$ Institute of Control Sciences of Russian Academy of Sciences
}

\begin{abstract}
In this paper, we discuss the problem of the pricing of American-style options in the exponential Lévy security market model. This model is typically incomplete, and we derive the explicit bounds of the interval of no arbitrage prices and the related optimal stopping moments for American put options and American call options in both finite and infinite horizon time. We consider a large class of Lévy processes.
\end{abstract}

Keywords: Incomplete market; Lévy process; American option; upper price; lower price; optimal stopping

2000 Mathematics Subject Classification: Primary 60G40; 60J75; 91B70

\section{Introduction}

In this paper we consider the traditional $(B, S)$-model of financial markets, in which a risky asset (stock) $S_{t}$ is defined as

$$
S_{t}=S_{0} \exp \left(X_{t}\right)
$$

with a constant initial price $S_{0}$ and a Lévy return process $X_{t}$. There are a lot of papers where the idea of Lévy distribution of returns is successfully based empirically (see Eberlein and Keller (1995), Barndorff-Nielsen (1998), Eberlein and Prause (2002), and Carr et al. (2002)). But the problem is that this model is usually incomplete. There typically exists a large set of risk-neutral measures instead of the unique risk-neutral measure in complete cases, for example in the Black-Scholes model, and a whole interval of no arbitrage prices goes here. The problem of pricing in incomplete markets is obviously to characterize this interval somehow.

When European options are discussed, there are many works, both in discrete and continuous time, on this topic. The upper and lower bounds of the interval of fair prices are derived. We refer to Rüschendorf (2002) and Gushchin and Mordecki (2002) for results on this topic in discrete time established by the Cox-Ross-Rubinstein model. Frey and Sin (1999) derived the bounds for the stochastic volatility model. Bellamy and Jeanblanc-Picqué (2000) obtained the trival upper and Black-Scholes lower prices of European call options for some special class of jump-diffusion processes. See also Henderson and Hobson (2003). Lévy processes are completely considered by Eberlein and Jacod (1997), for processes with vanishing continuous part, and by Jakubénas (2002), for other types of processes. On this topic, see also Cherny and Shiryaev (2002) and Selivanov (2004). For extensions of all mentioned results to the general semimartingale framework, see Gushchin and Mordecki (2002) for the 1-risky asset model and Bergenthum and Rüschendorf (2006) for the $d$-risky assets model.

For the case of American options, the results are much more limited. We can mention only the papers Bellamy and Jeanblanc-Picqué (2000) and Ekström (2006), which considered

Received 21 June 2006; revision received 11 December 2006.

* Postal address: Laboratory 38, Institute of Control Sciences, Russian Academy of Sciences, Profsoyuznaya 65, 117997 Moscow, Russia. Email address: roivanov@yahoo.com 
a jump-diffusion model with Poisson jump measure and obtained the bounds based on the American Black-Scholes pricing function for American options in finite time and for perpetual American options respectively.

This paper deals with American options. We consider both American put options and American call options in finite and infinite (i.e. perpetual options) horizon time models of exponential Lévy markets. A large class of Lévy processes is discussed; in particular, both purely discontinuous processes and the Lévy processes with nonnull Brownian part. The results are given in the form of the bounds of intervals of no arbitrage prices and also optimal stopping moments for them (see Theorem 1 and Theorem 2, below).

This paper is organized as follows. In Section 2, we introduce the necessary notation and present our results; in Section 3, we provide the proofs of our results.

\section{Results}

As we mentioned above, our model has prices of nonrisky and risky assets given as

$$
B_{t}=B_{0} \exp (r t) \quad \text { and } \quad S_{t}=S_{0} \exp \left(X_{t}\right)
$$

respectively, where $B_{0}$ and $S_{0}$ are constants, $r>0$ is a risk-free interest rate, and $X=\left(X_{t}\right)_{t \geq 0}$ is a Lévy process on some probability space with filtration $\left(\Omega, \mathcal{F},\left(\mathcal{F}_{t}\right)_{t \geq 0}, \mathrm{P}\right)$. We denote by $(b, c, v)_{H}$ the triplet of characteristics of the process $X$ taken with the truncation function $H(x)=x \mathbf{1}_{\{|x| \leq 1\}}$ (where $\mathbf{1}_{\{\cdot\}}$ denotes the indicator function), i.e.

$$
\mathrm{E}_{\mathrm{P}}\left[\exp \left(i \lambda X_{t}\right)\right]=\exp \left(t\left(i \lambda b-\frac{c \lambda^{2}}{2}+\int_{\mathbb{R}}\left(\mathrm{e}^{i \lambda x}-1-i \lambda H(x)\right) v(\mathrm{~d} x)\right)\right), \quad \lambda \in \mathbb{R},
$$

where $\mathrm{E}_{\mathrm{P}}$ denotes the expectation with respect to $\mathrm{P}, v(\{0\})=0$, and

$$
\int_{\mathbb{R}}\left(x^{2} \wedge 1\right) v(\mathrm{~d} x)<\infty .
$$

Note here that the representation (1) can also take place with any other truncation function $h=h(x)$, but the first component of the triplet changes. That is, for two truncation functions $h$ and $h^{\prime}$ we have the following for the first components of the triplets:

$$
b(h)-b\left(h^{\prime}\right)=\int_{\mathbb{R}}\left(h(x)-h^{\prime}(x)\right) v(\mathrm{~d} x) .
$$

Set

$$
\tilde{b}=b-\int_{\mathbb{R}} H(x) v(\mathrm{~d} x) ;
$$

we can see that $\tilde{b}$ is not defined here if the following equalities both hold at the same time:

$$
\int_{0}^{1} x v(\mathrm{~d} x)=\infty \text { and } \int_{-1}^{0} x v(\mathrm{~d} x)=-\infty .
$$

Also, we define a set of Lévy processes

$$
s^{+}=\left\{X \sim(b, 0, v)_{H}: v(-\infty, 0)=0,0<\int_{0}^{1} x v(\mathrm{~d} x)<\infty\right\} .
$$


If $X \in \delta^{+}$has the first characteristic with respect to the zero truncation function $\tilde{b} \geq 0$, then $X$ is a subordinator (see e.g. Sato (1999, Theorem 21.5)). We can understand $\delta^{+}$as a set of subordinators with drift. We also define

$$
s^{-}=\left\{-X: X \in 8^{+}\right\} .
$$

In contrast to European contingent claims, American contingent claims can be exercised at any moment of the life of a contract. For some $K>0$, the American call option has payoffs $f_{t}=\left(S_{t}-K\right)^{+}$, and the American put option has payoffs $f_{t}=\left(K-S_{t}\right)^{+}$. If the set $\mathcal{M}(\mathrm{P})$ of equivalent martingale measures is nonempty, then the upper (seller) and lower (buyer) prices of such option are

$$
\bar{V}=\sup _{Q \in \mathcal{M}(\mathrm{P})} \sup _{\tau \in \mathfrak{M}_{0}^{T}} B_{0} \mathrm{E}_{Q}\left[\frac{f_{\tau}}{B_{\tau}}\right]=\sup _{Q \in \mathcal{M}(\mathrm{P})} \sup _{\tau \in \mathfrak{M}_{0}^{T}} \mathrm{E}_{Q}\left[\mathrm{e}^{-r \tau} f_{\tau}\right]
$$

and

$$
\underline{V}=\inf _{Q \in \mathcal{M}(\mathrm{P})} \sup _{\tau \in \mathfrak{M}_{0}^{T}} \mathrm{E}_{Q}\left[\mathrm{e}^{-r \tau} f_{\tau}\right]
$$

respectively, where we use a 'martingale' no arbitrage concept introduced by Sin (1996) and Yan (1998). Here, $\mathfrak{M}_{0}^{T}$ denotes the set of all stopping moments $\tau \in[0, T]$ in the case of finite $T$ and the set of all $\tau \in[0, \infty)$ for perpetual options. On the definition of the low price, we refer to Harrison and Kreps (1979, Section 7) or Kramkov (1996, Theorem 3.3), the usual property $\underline{V}(f)=-\bar{V}(-f)$, and the fact that if there is a unique martingale measure $Q$, to obtain

$$
\underline{V}=\bar{V}=\sup _{\tau \in \mathfrak{M}_{0}^{T}} \mathrm{E}_{Q}\left[\mathrm{e}^{-r \tau} f\left(S_{\tau}\right)\right]
$$

We denote the upper and lower prices of the American put option in finite horizon time by

$$
\bar{V}_{\mathrm{p}} \text { and } \underline{V}_{\mathrm{p}},
$$

respectively, and we denote the upper and lower prices of the perpetual American put option by

$$
\bar{V}_{\mathrm{p}}^{\infty} \text { and } \underline{V}_{\mathrm{p}}^{\infty},
$$

respectively. For the American call option the equivalent terms are

$$
\bar{V}_{\mathrm{c}}, \quad \underline{V}_{\mathrm{c}}, \quad \bar{V}_{\mathrm{c}}^{\infty}, \quad \underline{V}_{\mathrm{c}}^{\infty} .
$$

Next, for a measure $Q \in \mathcal{M}(\mathrm{P})$, we set

$$
V(Q)=\sup _{\tau \in \mathfrak{M}_{0}^{T}} \mathrm{E}_{Q}\left[\mathrm{e}^{-r \tau} f_{\tau}\right]
$$

for $T \in[0, \infty]$. We say that a stopping moment $\tau_{Q}$ is the $V(Q)$-optimal stopping moment if

$$
V(Q)=\mathrm{E}_{Q}\left[\exp \left(-r \tau_{Q}\right) f_{\tau_{Q}}\right] .
$$

Note that a $\tau_{Q}$ defined in this way is the optimal stopping moment for the buyer of the option (see, for example, Shiryaev (1999, Section VI.2c)). We denote the optimal stopping moments for the related upper and lower prices of put options and call options by

$$
\bar{\tau}_{\mathrm{p}}^{T}, \quad \underline{\tau}_{\mathrm{p}}^{T}, \quad \bar{\tau}_{\mathrm{p}}^{\infty}, \quad \underline{\tau}_{\mathrm{p}}^{\infty}, \quad \bar{\tau}_{\mathrm{c}}^{T}, \quad \underline{\tau}_{\mathrm{c}}^{T}, \quad \bar{\tau}_{\mathrm{c}}^{\infty}, \quad \underline{\tau}_{\mathrm{c}}^{\infty} .
$$


Theorem 1. If $X$ is not in $8^{+}$or $8^{-}$and is not a Brownian motion with drift, then

$$
\bar{V}_{\mathrm{p}}=\bar{V}_{\mathrm{p}}^{\infty}=K \text { and } \bar{V}_{\mathrm{c}}=\bar{V}_{\mathrm{c}}^{\infty}=S_{0} .
$$

The optimal stopping moments $\bar{\tau}_{\mathrm{p}}^{T}$ and $\bar{\tau}_{\mathrm{p}}^{\infty}$ do not exist. However, for any $\delta>0$, there exists the $\delta$-optimal stopping moment

$$
\bar{\tau}_{\mathrm{p}}^{T}(\delta)=\bar{\tau}_{\mathrm{p}}^{\infty}(\delta)=\frac{1}{r} \log \frac{K}{K-\delta} \quad(\mathrm{P} \text {-almost surely) }
$$

such that

$$
\sup _{Q \in \mathcal{M}(\mathrm{P})} \mathrm{E}_{Q}\left[\exp \left(-r \bar{\tau}_{\mathrm{p}}^{T}(\delta)\right)\left(K-S_{\bar{\tau}_{\mathrm{p}}^{T}(\delta)}\right)^{+}\right] \geq K-\delta
$$

We have

$$
\bar{\tau}_{\mathrm{c}}^{T}=T
$$

and, for any $\Delta>0$,

$$
\sup _{Q \in \mathcal{M}(\mathrm{P})} \mathrm{E}_{Q}\left[\mathrm{e}^{-r \Delta}\left(S_{\Delta}-K\right)^{+}\right]=S_{0} .
$$

Note that (denoting the upper price of the European put option by $\bar{V}_{\mathrm{Ep}}$ ) we have, from the 'call-put parity' and the results of Eberlein and Jacod (1997) and Jakubénas (2002), that $\bar{V}_{\mathrm{Ep}}=\mathrm{e}^{-r T} K<\bar{V}_{\mathrm{p}}$ if $r>0$. As usual, European call options and American call options are tallied.

Theorem 2. Assume that the triplet of characteristics $(b, 0, v)_{H}$ of $X$ satisfies at least one of the following conditions:

(i) $\nu(0, \varepsilon)>0$, for any $\varepsilon>0$ and $-\infty<\tilde{b}-r<0$, or $\nu(-\varepsilon, 0)>0$, for any $\varepsilon>0$ and $0<\tilde{b}-r<\infty$,

(ii) $\tilde{b}=\infty$ or $\tilde{b}=-\infty$,

(iii) $\tilde{b}$ is not defined.

Then

$$
\begin{gathered}
\underline{V}_{\mathrm{p}}=\underline{V}_{\mathrm{p}}^{\infty}=\left(K-S_{0}\right)^{+}, \\
\underline{\tau}_{\mathrm{p}}^{T}=\underline{\tau}_{\mathrm{p}}^{\infty}=0
\end{gathered}
$$

and

$$
\begin{gathered}
\underline{V}_{\mathrm{c}}=\left(S_{0}-\mathrm{e}^{-r T} K\right)^{+}, \quad \underline{V}_{\mathrm{c}}^{\infty}=S_{0}, \\
\underline{\tau}_{\mathrm{c}}^{T}=T .
\end{gathered}
$$

The optimal stopping moment $\underline{\tau}_{\mathrm{c}}^{\infty}$ does not exist, but for any $\delta>0$ there exists the $\delta$-optimal stopping moment

$$
\underline{\tau}_{\mathrm{c}}^{\infty}(\delta)=\frac{1}{r} \log \frac{K}{\delta} \quad(\mathrm{P} \text {-almost surely }),
$$

such that

$$
\inf _{Q \in \mathcal{M}(\mathrm{P})} \mathrm{E}_{Q}\left[\exp \left(-r \underline{\tau}_{\mathrm{c}}^{\infty}(\delta)\right)\left(S_{\underline{\tau}_{\mathrm{c}}^{\infty}(\delta)}-K\right)^{+}\right] \geq S_{0}-\delta
$$


Let the assumptions of both Theorem 1 and Theorem 2 be satisfied for the triplet of characteristics of $X_{t}$. Then we have for American put options a wider interval of fair prices $\left\langle\left(K-S_{0}\right)^{+}, K\right\rangle$, instead of $\left\langle\left(\mathrm{e}^{-r T} K-S_{0}\right)^{+}, \mathrm{e}^{-r T} K\right\rangle$ for European put options. For perpetual American call options, the interval of fair prices becomes the point $S_{0}$. And for perpetual American put options we have the same interval as in finite horizon time. Moreover, on the optimal stopping we can see that if a holder of an American put option wants to guarantee themselves, they have to stop at zero time. And for the American call option, we need to continue to observe it for as long as possible.

Remark 1. The price of a perpetual American option can be defined in a different way as well. In this case, the set $\overline{\mathfrak{M}}_{0}^{\infty}$ of all stopping moments $\tau \in[0, \infty]$ is considered instead of $\mathfrak{M}_{0}^{\infty}$ (see Shiryaev (1999, Section VIII.2) and Ivanov (2005)). Here, the event $\{\tau=\infty\}$ means that the option is not exercised and, for example, the lower price of an American put option is

$$
\underline{V}_{\overline{\mathrm{p}}}^{\infty}=\inf _{Q \in \mathcal{M}(\mathrm{P})} \sup _{\tau \in \overline{\mathfrak{M}}_{0}^{\infty}} \mathrm{E}_{Q}\left[\mathrm{e}^{-r \tau} f_{\tau} \mathbf{1}_{\{\tau<\infty\}}\right]
$$

Looking at the results of Theorems 1 and 2, we can observe that it could happen that $\underline{V}_{\overline{\mathrm{p}}}^{\infty}>\underline{V}_{\mathrm{p}}^{\infty}$, since $\mathfrak{M}_{0}^{\infty} \subset \overline{\mathfrak{M}}_{0}^{\infty}$. In fact, $\underline{V}_{\overline{\mathrm{p}}}^{\infty}=\underline{V}_{\mathrm{p}}^{\infty}$ (see (11), below).

Remark 2. In this paper we use a martingale no arbitrage concept. However, if we impose the set of admissible strategies to appropriate restrictions (see Delbaen and Schachermayer (1998) and Shiryaev (1999)) and substitute the set $\mathcal{M}(\mathrm{P})$ by the set of equivalent $\sigma$-martingale measures, the results of Theorem 1 and Theorem 2 do not change. In fact, in the proofs we obtain the bounds of intervals of fair prices and then derive sequences of martingale measures which give the sequences converging to these bounds. Obviously, these sequences of measures are also $\sigma$-martingales, and the results follow.

Note finally that if the interest rate $r=0$, then both processes $\left(S_{t}-K\right)^{+}$and $\left(K-S_{t}\right)^{+}$are submartingales with respect to any $Q \in \mathcal{M}(\mathrm{P})$ by Jensen's inequality, and the American call option and the American put option coincide with the appropriate European options.

\section{Proofs}

For simplicity, we set $Y_{t}=X_{t}-r t$. For a sequence of random variables $F_{n}$ we denote its weak convergence to $F$ by $F_{n} \stackrel{\mathrm{w}}{\rightarrow} F$.

\subsection{Proof of Theorem 1}

First we prove the case of a put option in the finite interval $[0, T]$. For any $Q \in \mathcal{M}(\mathrm{P})$ and any $\tau \in \mathfrak{M}_{0}^{T}$, we have

$$
\mathrm{E}_{Q}\left[\mathrm{e}^{-r \tau}\left(K-S_{\tau}\right)^{+}\right] \leq K
$$

therefore,

$$
\bar{V}_{\mathrm{p}} \leq K
$$

Set $\tau=\varepsilon$, where $\varepsilon>0$ is an arbitrary number. Let us construct a sequence of measures $\left\{Q_{n}\right\} \in \mathcal{M}(\mathrm{P})$ such that

$$
\mathrm{E}_{Q_{n}}\left[\mathrm{e}^{-r \varepsilon}\left(K-S_{\varepsilon}\right)^{+}\right] \rightarrow \mathrm{e}^{-r \varepsilon} K \quad \text { as } n \rightarrow \infty .
$$

We now consider the various cases of the triplet $(b, c, v)_{H}$ of $Y_{t}$. 
Case I. Suppose that $v(-\infty, 0)>0$ and $v(0, \infty)>0$. Here, $\mathcal{M}(\mathrm{P}) \neq \varnothing$ (see e.g. Selivanov (2004, Theorem 3.2)). With no restriction of generality, we suppose that the initial measure $\mathrm{P}$ is a martingale, i.e. $\mathrm{P} \sim(b, c, v)_{H}$. Then we obtain

$$
b+\frac{c}{2}+\int_{\mathbb{R}}\left(\mathrm{e}^{x}-1-H(x)\right) v(\mathrm{~d} x)=0
$$

(see Shiryaev (1999, Theorem 3, Section VII.3c)).

For some $a>0$, we have $v(a, \infty)>0$ and $v(-\infty,-a)>0$. If $a>1$, set $a=1$. We rewrite (4) as

$$
b_{a}+\frac{c}{2}+\int_{\mathbb{R}}\left(\mathrm{e}^{x}-1-H_{a}(x)\right) v(\mathrm{~d} x),
$$

with

$$
\begin{aligned}
b_{a} & =b-\int_{[-1,1] \backslash[-a, a]} x \mathcal{d}(\mathrm{d} x), \\
H_{a}(x) & =x \mathbf{1}_{\{|x| \leq a\}} .
\end{aligned}
$$

We define a constant $\theta$ by

$$
\theta=\frac{\int_{a}^{\infty}\left(\mathrm{e}^{x}-1\right) v(\mathrm{~d} x)}{\int_{-\infty}^{-a}\left(1-\mathrm{e}^{x}\right) v(\mathrm{~d} x)}
$$

and set

$$
v_{n}=v+\left.n v\right|_{(a, \infty)}+\left.\theta n v\right|_{(-\infty,-a)} .
$$

The new measure $Q_{n} \sim\left(b_{a}, c, v_{n}\right)_{H_{a}}$ is a martingale for the process $Y_{t}$ for any $n \geq 1$ since

$$
b_{a}+\frac{c}{2}+\int_{\mathbb{R}}\left(\mathrm{e}^{x}-1-H_{a}(x)\right) v_{n}(\mathrm{~d} x)=0 .
$$

Let $Y^{n}$ be the process $Y$ under $Q_{n}$. By Sato (1999, Theorem 19.2), we can decompose $Y^{n}$ as the sum of two independent Lévy processes, $Y^{n, 1}$ and $Y^{n, 2}$, with triplets of characteristics $\left(b_{a}, c, v\right)_{H_{a}}$ and $\left(0,0,\left.n v\right|_{(a, \infty)}+\left.\theta n v\right|_{(-\infty,-a)}\right)_{0}$, respectively. We see that $Y^{n, 1}$ has the same distribution under $Q_{n}$ as $Y$ has under P, and

$$
\mathrm{E}_{Q_{n}}\left[\exp \left(\frac{Y_{\varepsilon}^{n, 2}}{2}\right)\right]=\exp \left(n \varepsilon\left[\theta \int_{-\infty}^{-a}\left(\mathrm{e}^{x / 2}-1\right) v(\mathrm{~d} x)+\int_{a}^{\infty}\left(\mathrm{e}^{x / 2}-1\right) v(\mathrm{~d} x)\right]\right) .
$$

From (5), (6), and the inequalities $\mathrm{e}^{x}-1>2\left(\mathrm{e}^{x / 2}-1\right)$ and $2\left(1-\mathrm{e}^{x / 2}\right)>1-\mathrm{e}^{x}$ for $x>0$, we conclude that

$$
\mathrm{E}_{Q_{n}}\left[\exp \left(\frac{Y_{\varepsilon}^{2}}{2}\right)\right]=\exp (n \varepsilon \xi),
$$

where $\xi<0$. It follows from (7) that $Y_{\varepsilon}^{n, 2} \stackrel{\mathrm{w}}{\rightarrow}-\infty$ and, therefore, $Y_{\varepsilon}^{n} \stackrel{\mathrm{w}}{\rightarrow}-\infty$ as $n \rightarrow \infty$. Therefore, (3) holds for the constructed sequence $\left\{Q_{n}\right\}$.

Case II. Suppose that $v$ is concentrated on $(0, \infty), c=0$, and $\int_{0}^{1} x v(\mathrm{~d} x)=\infty$. Let $Y \sim$ $(b, 0, v)_{H}$ under the initial measure P. Choose $0<a<1, a \in \operatorname{supp} v$. Define a sequence $\varepsilon_{n} \rightarrow 0$ satisfying

$$
b-\int_{\varepsilon_{n}}^{1} x v(\mathrm{~d} x)=-f_{n} \leq-n \quad \text { for } n \in \mathbb{N} .
$$


Let us construct a sequence $\left\{Q_{n}\right\} \in \mathcal{M}(\mathrm{P}), Q_{n} \sim\left(b_{n}, c, v_{n}\right)_{H}$, setting

$$
\begin{aligned}
& \left.v_{n}\right|_{\left[0, \varepsilon_{n}\right]}=\left.v\right|_{\left[0, \varepsilon_{n}\right)}, \\
& \left.v_{n}\right|_{\mathbb{R} \backslash\left\{\left[0, \varepsilon_{n}\right] \cup[a-1 / n, a+1 / n]\right\}} \text { such that } \int_{\mathbb{R} \backslash\left\{\left[0, \varepsilon_{n}\right] \cup[a-1 / n, a+1 / n]\right\}}\left(\mathrm{e}^{x}-1\right) v_{n}(\mathrm{~d} x)=\frac{1}{n}, \\
& \left.v_{n}\right|_{[a-1 / n, a+1 / n]}=\left.\theta_{n} \nu\right|_{(a-1 / n, a+1 / n)},
\end{aligned}
$$

where $\theta_{n}$ is defined by (4), which we rewrite as

$$
b_{n}+\int_{\mathbb{R}}\left(\mathrm{e}^{x}-1-H(x)\right) v_{n}(\mathrm{~d} x)=0,
$$

here. From (8), using Sato (1999, Equation (33.4)), we have

$$
\theta_{n}=\frac{f_{n}+\alpha_{n}}{\int_{a-1 / n}^{a+1 / n}\left(\mathrm{e}^{x}-1\right) v(\mathrm{~d} x)}
$$

where $\alpha_{n} \rightarrow 0$ as $n \rightarrow \infty$.

Then, for a fixed $u, 0<u<1$, we have

$$
\mathrm{E}_{Q_{n}}\left[\exp \left(u Y_{\varepsilon}\right)\right]=\exp \left(\varepsilon f_{n}\left(-u+\bar{o}(1)+\frac{\mathrm{e}^{u a}-1}{\mathrm{e}^{a}-1}\right)\right) \quad \text { as } n \rightarrow \infty .
$$

A function $g(u)=-u \mathrm{e}^{a}+u+\mathrm{e}^{u a}-1$ has $g(0)=0$ and $g^{\prime}(0)=-\mathrm{e}^{a}+1+a<0$; hence, there exists $u$ such that $\mathrm{E}_{Q_{n}}\left[\exp \left(u Y_{\varepsilon}\right)\right] \rightarrow 0$ as $n \rightarrow \infty$. Case II can be completed in a similar way to Case I.

Case III. Suppose that $v$ is concentrated on $(0, \infty)$ and $c>0$. As in Case I we mean that $\mathrm{P}$ is a martingale, $\mathrm{P} \sim(b, c, v)_{H}$. Take $a \in \operatorname{supp} v$ and choose a sequence of numbers $\left\{\theta_{n}\right\}$ in such a way so that

$$
\theta_{n} v\left\{\left[a-\frac{1}{n}, a+\frac{1}{n}\right]\right\}=n
$$

Set

$$
\begin{aligned}
& v_{n}=v+\left.\theta_{n} v\right|_{[a-1 / n, a+1 / n]}, \\
& b_{n}=b-\theta_{n} \int_{a-1 / n}^{a+1 / n}\left(\mathrm{e}^{x}-1-H(x)\right) v(\mathrm{~d} x),
\end{aligned}
$$

since $c>0$. The process $Y^{n}$ taken under $Q_{n} \sim\left(b_{n}, c, v_{n}\right)_{H}$ can be decomposed into the sum of two independent Lévy processes, $Y^{n, 1} \sim(b, c, v)_{H}$ and $Y^{n, 2} \sim\left(b_{n}-b, c, v_{n}-v\right)_{H}$. Now, we have

$$
\mathrm{E}\left[\exp \left(\frac{Y_{\varepsilon}^{n, 2}}{2}\right)\right]=\exp \left(-\varepsilon \theta_{n} \int_{a-1 / n}^{a+1 / n}\left(\frac{1}{2} \mathrm{e}^{x}-\mathrm{e}^{x / 2}+\frac{1}{2}\right) v(\mathrm{~d} x)\right) .
$$

Since $g(x)=\frac{1}{2} \mathrm{e}^{x}-\mathrm{e}^{x / 2}+\frac{1}{2}>0$ for any $x>0$, we have $\mathrm{E}\left[\exp \left(Y_{\varepsilon}^{n, 2} / 2\right)\right] \rightarrow 0$ and we can therefore deduce that $Y_{\varepsilon}^{n} \stackrel{\mathrm{w}}{\rightarrow}-\infty$ in a similar way to in Case I.

Case IV. If $v$ is concentrated on $(0, \infty), \int_{0}^{1} x v(\mathrm{~d} x)<\infty$, and $c=0$, we have the case of a subordinator with drift.

Case V. If $v=0$ we have a Brownian motion with drift. 
The cases, where $v$ is concentrated on $(-\infty, 0)$, are proved in a similar way to Cases II-V. From the arbitrariness of $\varepsilon$, we obtain our theorem for put options in finite horizon time.

For perpetual put options we obviously have

$$
\bar{V}_{\mathrm{p}}^{\infty} \leq K \quad \text { and } \quad \bar{V}_{\mathrm{p}}^{\infty} \geq \bar{V}_{\mathrm{p}}=K
$$

therefore, $\bar{V}_{\mathrm{p}}^{\infty}=K$.

Finally, for call options we have the same proof as for put options, substituting the usual argument of boundness of $\left(K-S_{t}\right)^{+}$by Jakubénas (2002, Lemma 10). The part of the theorem concerning optimal stopping moments clearly follows from (3).

\subsection{Proof of Theorem 2}

First, we prove Theorem 2 for put options. We have for the stopping moment $\tau_{0}=0$ (P-almost surely)

$$
\begin{aligned}
\underline{V}_{\mathrm{p}} & =\inf _{Q \in \mathcal{M}(\mathrm{P})} \sup _{\tau \in \mathfrak{M}_{0}^{T}} \mathrm{E}_{Q}\left[\mathrm{e}^{-r \tau}\left(K-S_{\tau}\right)^{+}\right] \\
& \geq \inf _{Q \in \mathcal{M}(\mathrm{P})} \mathrm{E}_{Q}\left[\exp \left(-r \tau_{0}\right)\left(K-S_{\tau_{0}}\right)^{+}\right] \\
& =\left(K-S_{0}\right)^{+} .
\end{aligned}
$$

On the other hand,

$$
\begin{aligned}
\underline{V}_{\mathrm{p}} & \leq \inf _{Q \in \mathcal{M}(\mathrm{P})} \sup _{\tau \in \mathfrak{M}_{0}^{T}} \mathrm{E}_{Q}\left[\left(K-\mathrm{e}^{-r \tau} S_{\tau}\right)^{+}\right] \\
& =\inf _{Q \in \mathcal{M}(\mathrm{P})} \mathrm{E}_{Q}\left[\left(K-\mathrm{e}^{-r T} S_{T}\right)^{+}\right]
\end{aligned}
$$

since $\left(K-\mathrm{e}^{-r t} S_{t}\right)^{+}$is submartingale by Jensen's inequality. We construct a sequence of measures $\left\{Q_{n}\right\} \in \mathcal{M}(\mathrm{P})$ such that

$$
\mathrm{E}_{Q_{n}}\left[\left(K-\mathrm{e}^{-r T} S_{T}\right)^{+}\right] \rightarrow\left(K-S_{0}\right)^{+} \text {as } n \rightarrow \infty .
$$

We now discuss the various cases of the triplet of characteristics of $Y_{t}$.

Case I. Suppose that $v((0, \varepsilon))>0$, for any $\varepsilon>0$, and $-\infty<\tilde{b}<0$. That is, $\mathrm{P} \sim(b, 0, v)_{H}$. Here, we construct a sequence of equivalent martingale measures $Q_{n} \sim\left(b_{n}, 0, v_{n}\right)_{H}$ such that

$$
\mathrm{E}_{Q_{n}}\left[\left(K-\mathrm{e}^{-r T} S_{T}\right)\right]=\mathrm{E}\left[\left(K-S_{0} \exp \left(\frac{1}{n} N\left(\lambda_{1 / n} T\right)+\tilde{b} T\right)\right)^{+}\right],
$$

where $\lambda_{1 / n}=-\tilde{b} /\left(\mathrm{e}^{1 / n}-1\right)$ and $N(\lambda)$ denotes a Poisson process with intensity $\lambda$. Then we can immediately use Jakubénas (2002, Lemma 4) to obtain (9).

For a fixed $n>0,1 / n \in \operatorname{supp} v$, we define, for all sufficient large $k$, a Lévy measure $v_{n, k}$ by

$$
\int_{\mathbb{R} \backslash[1 / n-1 / k, 1 / n+1 / k]}\left(\left|\mathrm{e}^{x}-1\right|+|H(x)|\right) v_{n, k}(\mathrm{~d} x)=\frac{1}{k}
$$

and

$$
\left.v_{n, k}\right|_{[1 / n-1 / k, 1 / n+1 / k]}=\left.\theta_{n, k} \nu\right|_{[1 / n-1 / k, 1 / n+1 / k]},
$$


where $\theta_{n, k}$ is defined due to the condition of Jakubénas (2002, Equation (33.4)) on an equivalent martingale measure $Q_{n, k} \sim\left(b_{n, k}, 0, v_{n, k}\right)_{H}$ :

$$
b-\int_{\mathbb{R}} H(x) v(\mathrm{~d} x)+\int_{\mathbb{R}}\left(\mathrm{e}^{x}-1\right) v_{n, k}(\mathrm{~d} x)=0 ;
$$

hence,

$$
\theta_{n, k}=-\frac{\tilde{b}+\alpha_{k}}{\int_{1 / n-1 / k}^{1 / n+1 / k}\left(\mathrm{e}^{x}-1\right) v(\mathrm{~d} x)},
$$

where $\alpha_{k} \rightarrow 0$ as $k \rightarrow \infty$.

From (10) we obtain

$$
b_{n, k} \rightarrow \tilde{b}+\lambda_{1 / n} H\left(\frac{1}{n}\right)=: b_{n},
$$

where $\lambda_{1 / n}=-\tilde{b} /\left(\mathrm{e}^{1 / n}-1\right)$, and

$$
\left(x^{2} \wedge 1\right) v_{n, k}(\mathrm{~d} x) \stackrel{\mathrm{W}}{\rightarrow}\left(x^{2} \wedge 1\right) \lambda_{1 / n} \delta_{1 / n}(\mathrm{~d} x)=\left(x^{2} \wedge 1\right) v_{n}(\mathrm{~d} x) \quad \text { as } k \rightarrow \infty .
$$

Therefore, from Jacod and Shiryaev (1987, Theorem VII.2.9), we have

$$
\begin{aligned}
& \mathrm{E}_{Q_{n, k}}\left[\left(K-S_{0} \exp \left(Y_{T}\right)\right)^{+}\right] \\
& \quad \rightarrow \mathrm{E}_{Q_{n}}\left[\left(K-\mathrm{e}^{-r T} S_{T}\right)^{+}\right]=\mathrm{E}\left[\left(K-S_{0} \exp \left(\frac{1}{n} N\left(\lambda_{1 / n} T\right)+\tilde{b} T\right)\right)^{+}\right] \text {as } k \rightarrow \infty .
\end{aligned}
$$

Case II. Suppose that $\tilde{b}=-\infty$ and $\mathrm{P} \sim(b, 0, v)_{H}$. We can choose a sequence $0<\varepsilon_{n} \rightarrow 0$ such that

$$
\int_{\varepsilon_{n}}^{1 / n} x \nu(\mathrm{d} x)=\int_{1 / n}^{1} x v(\mathrm{~d} x) .
$$

Set

$$
\begin{aligned}
& \left.v_{n}\right|_{\left[-1 / n, \varepsilon_{n}\right]}=\left.v\right|_{\left[-1 / n, \varepsilon_{n}\right]}, \\
& \left.v_{n}\right|_{\mathbb{R} \backslash[-1 / n, 1 / n]} \text { such that } \int_{\mathbb{R} \backslash[-1 / n, 1 / n]}\left(\left|\mathrm{e}^{x}-1\right|+|H(x)|\right) v_{n}(\mathrm{~d} x)=\frac{1}{n}, \\
& \left.v_{n}\right|_{\left(\varepsilon_{n}, 1 / n\right]}=\left.\theta_{n} v\right|_{{ }_{\left.\varepsilon_{n}, 1 / n\right]} .}
\end{aligned}
$$

From the martingale condition on this measure $Q_{n} \sim\left(b_{n}, 0, v_{n}\right)_{H}$, it is not difficult to obtain

$$
\theta_{n} \rightarrow 2 \text { as } n \rightarrow \infty
$$

therefore, $\theta_{n}$ is bounded. Finally, we have

$$
\left(x^{2} \wedge 1\right) v_{n}(\mathrm{~d} x) \stackrel{\mathrm{w}}{\rightarrow} 0 \quad \text { and } \quad b_{n}=-\int_{\mathbb{R}}\left(\mathrm{e}^{x}-1-H(x)\right) v_{n}(\mathrm{~d} x) \rightarrow 0 \quad \text { as } n \rightarrow \infty .
$$

Therefore, $Y_{T}^{n} \stackrel{\mathrm{w}}{\rightarrow} 0$ and (9) follows.

The cases which deal with $\left.v\right|_{(-\infty, 0)}$ are considered in a similar way to Cases I-II. 
Case III. Suppose that $\tilde{b}$ is not defined and $\mathrm{P} \sim(b, 0, v)_{H}$. Then we can write $Y$ under $\mathrm{P}$ as the sum of two independent Lévy processes with triplets of characteristics $\left(b, 0,\left.v\right|_{(-\infty,-1) \cup[0,1]}\right)_{H}$ and $\left(0,0,\left.v\right|_{[-1,0] \cup(1, \infty)}\right)_{H}$. We can use to them Case II and the symmetric of $\tilde{b}=\infty$. Under constructed $\left\{Q_{n}\right\}$, we have

$$
Y_{T}^{n, 1} \stackrel{\mathrm{w}}{\rightarrow} 0 \quad \text { and } \quad Y_{T}^{n, 2} \stackrel{\mathrm{w}}{\rightarrow} 0
$$

for these two processes. Therefore, $Y_{T}^{n} \stackrel{\mathrm{w}}{\rightarrow} 0$ and we again have (9).

For perpetual put options we have, for every $Q \in \mathcal{M}(\mathrm{P})$ and $\tau \in \mathfrak{M}_{0}^{\infty}$,

$$
\begin{aligned}
\mathrm{E}_{Q}\left[\mathrm{e}^{-r \tau}\left(K-S_{\tau}\right)^{+}\right] & =\mathrm{E}_{Q}\left[\mathbf{1}_{\{\tau \leq T\}} \mathrm{e}^{-r \tau}\left(K-S_{\tau}\right)^{+}\right]+\mathrm{E}_{Q}\left[\mathbf{1}_{\{\tau>T\}} \mathrm{e}^{-r \tau}\left(K-S_{\tau}\right)^{+}\right] \\
& \leq \mathrm{E}_{Q}\left[\mathrm{e}^{-r(\tau \wedge T)}\left(K-S_{\tau \wedge T}\right)^{+}\right]+\mathrm{e}^{-r T} K
\end{aligned}
$$

for any $T \in \mathbb{R}^{+}$.

For any $\varepsilon>0$, we can choose $T$ such that $\mathrm{e}^{-r T} K<\varepsilon$. Using the result for put options in bounded intervals on $[0, T]$, we obtain

$$
\underline{V}_{\mathrm{p}}^{\infty}=\left(K-S_{0}\right)^{+} .
$$

For call options we have the same proof as for put options, substituting the argument of boundness of $\left(K-S_{t}\right)^{+}$by Jakubénas (2002, Lemma 10) and keeping in mind the submartingale property $\mathrm{e}^{-r t}\left(S_{t}-K\right)^{+}$.

For perpetual call options we have

$$
\underline{V}_{\mathrm{c}}^{\infty} \geq\left(S_{0}-\mathrm{e}^{-r T} K\right)^{+}
$$

for any $T \leq 0$, and, since $\underline{V}_{\mathrm{c}}^{\infty} \leq \bar{V}_{\mathrm{c}}^{\infty}$, we obtain

$$
\underline{V}_{\mathrm{c}}^{\infty}=S_{0} .
$$

Finally, the form of optimal stopping moments follows from (2), the values of the lower prices, and (12).

\section{Acknowledgements}

I would like to thank A. S. Cherny and A. N. Shiryaev for valuable consideration and helpful remarks.

\section{References}

Barndorff-Nielsen, O. (1998). Processes of normal inverse Gaussian type. Finance Stoch. 2, 41-68.

Bellamy, N. and Jeanblanc-Picqué, M. (2000). Incompleteness of markets driven by a mixed diffusion. Finance Stoch. 4, 209-222.

Bergenthum, J. AND RÜschendorf, L. (2006). Comparison of option prices in semimartingale models. Finance Stoch. 10, 222-249.

Carr, P., Geman, H., Madan, D. And Yor, M. (2002). The fine structure of asset returns: an empirical investigation. J. Business 75, 305-332.

Cherny, A. S. And Shiryaev, A. N. (2002). Change of time and measure for Levy processes. (Lectures from the Summer School 'From Lévy processes to semimartingales - recent theoretical developments and applications to finance' (Aarhus, August 2002)).

Delbaen, F. And Schachermayer, W. (1998). The fundamental theorem of asset pricing for unbounded stochastic processes. Math. Ann. 312, 215-250.

EbERLEIn, E. AND JACOD, J. (1997). On the range of options prices. Finance Stoch. 1, 131-140. 
Eberlein, E. ANd Keller, U. (1995). Hyperbolic distributions in finance. Bernoulli 1, 281-299.

Eberlein, E. ANd Prause, K. (2002). The generalized hyperbolic model: financial derivatives and risk measures. In Mathematical Finance-Bachelier Congress, 2000 (Paris), Springer, Berlin, pp. 245-267.

Eкström, E. (2006). Bounds for perpetual American option prices in a jump diffusion model. J. Appl. Prob. 43, $867-873$

Frey, R. AND Sin, C. A. (1999). Bounds on European option prices under stochastic volatility. Math. Finance 9, 97-116.

Gushchin, A. A. AND Mordecki, E. (2002). Bounds on option prices for semimartingale market models. Proc. Steklov Inst. Math. 237, 73-113.

HARRISON, M. AND KREPS, D. (1979). Martingales and arbitrage in multiperiod securities markets. J. Econom. Theory 20, 381-408.

Henderson, V. And Hobson, D. G. (2003). Coupling and option price comparisons in a jump-diffusion model. Stoch. Stoch. Reports 75, 79-101.

Ivanov, R. V. (2005). Discrete approximation of finite-horizon American-style options. Lithuanian Math. J. 45, $525-536$.

JACOD, J. AND ShIRYAEv, A. N. (1987). Limit Theorems for Stochastic Processes. Springer, Berlin.

JAKUBÉNAS, P. (2002). On option pricing in certain incomplete markets. Proc. Steklov Math. Inst. 237, 123-142.

Kramkov, D. (1996). Optional decomposition of supermartingales and hedging contingent claims in incomplete security markets. Prob. Theory Relat. Fields 105, 459-479.

RüSCHENDORF, L. (2002). On upper and lower prices in discrete-time models. Proc. Steklov Inst. Math. 237, $134-139$.

Sato, K.-I. (1999). Lévy Processes and Infinitely Divisible Distributions. Cambridge University Press.

Selivanov, A. (2004). On martingale measures in exponential Lévy models. Theory Prob. Appl. 49, 317-334.

Shiryaev, A. N. (1999). Essentials of Stochastic Finance. Facts, Models, Theory. World Scientific, River Edge, NJ.

SIN, C. A. (1996). Strictly local martingales and hedge ratios in stochastic volatility models. Doctoral Thesis, Cornell University.

YAN, J. A. (1998). A new look at the fundamental theorem of asset pricing. J. Korean Math. Soc. 35, 659-673. 